\title{
Calibration Techniques and Strategies for the Present and Future LHC Electromagnetic Calorimeters
}

\author{
M. Aleksa on behalf of the ATLAS and CMS collaborations \\ CERN, \\ Geneva 23, 1211 Geneva, Switzerland \\ E-mail: martin.aleksa@cern.ch
}

Aвstract: This document describes the different calibration strategies and techniques applied by the two general purpose experiments at the LHC, ATLAS and CMS, and discusses them underlining their respective strengths and weaknesses from the view of the author. The resulting performances of both calorimeters are described and compared on the basis of selected physics results. Future upgrade plans for High Luminosity LHC (HL-LHC) are briefly introduced and planned calibration strategies for the upgraded detectors are shown.

\section{Introduction}

The ATLAS [1] and CMS [2] detectors are multipurpose particle physics experiments with forwardbackward symmetric cylindrical geometry at the CERN Large Hadron Collider (LHC). Both experiments optimized the electromagnetic calorimeters according to the physics requirements given by a few benchmark channels, such as the discovery of the Higgs boson (e.g. $\mathrm{H} \rightarrow \gamma \gamma$, $\mathrm{H} \rightarrow \mathrm{ZZ}^{(*)} \rightarrow 4 \mathrm{e}^{ \pm}, \mathrm{H} \rightarrow \mathrm{WW}^{(*)} \rightarrow \mathrm{e} v \mathrm{e} v$ ), precision measurements such as the mass of the $\mathrm{W}$ boson and the discovery of physics beyond the Standard Model (e.g. decays of heavy Z' and W' bosons with masses up to $6 \mathrm{TeV}$ ). In order to exploit the full physics potential, the electromagnetic calorimeters must be able to measure resonances in the diphoton invariant mass spectrum like e.g. a $\sim 125 \mathrm{GeV}$ Higgs boson with better than $1 \%$ precision and measure electrons and photons in a large energy range from $5 \mathrm{GeV}$ to $5 \mathrm{TeV}$ with high resolution and per-mill level linearity. For electron measurements between the $\mathrm{Z} \rightarrow$ ee peak and electrons from $\mathrm{W}$ decays (electron energy between 20 and $60 \mathrm{GeV}$ ), a linearity of better than $10^{-4}$ is required. In the following, the two electromagnetic calorimeters and their calibration strategies will be described in greater detail. 


\section{Layout of the Electromagnetic Calorimeters}

\subsection{ATLAS}

The ATLAS electromagnetic calorimeter is a lead-liquid argon (LAr) sampling calorimeter with accordion geometry. It is described in detail in Ref. [1] and [3]. It is divided into a barrel region, covering a pseudorapidity ${ }^{1}|\eta|<1.475$, and two end-caps covering $1.35<|\eta|<3$.2. The width of the active LAr gaps is $2 \mathrm{~mm}$ in the barrel and ranges from $1.2 \mathrm{~mm}$ to $2.7 \mathrm{~mm}$ in the endcap. The calorimeter is segmented into three longitudinal layers for $|\eta|<2.5$. The first layer, around 4-5 radiation lengths thick, is finely segmented in the $\eta$ direction to provide $\gamma-\pi^{0}$ separation and photon direction measurement. For instance, the cell size in $\eta$ is 0.003125 in the barrel region. The second layer collects most of the shower energy and has a granularity of $\Delta \eta \times \Delta \varphi=0.025 \times \pi / 128$ for $|\eta|<2.5$. The "photon pointing" measurement aligns the measurements from the first and the second layer and extrapolates the photon trajectory to the interaction region. The last layer is used to correct for leakage behind the electromagnetic calorimeter. All layers together correspond to more than 22 radiation lengths. In addition a thin presampler (PS) layer is located between the cryostat and the calorimeter, covering the region $|\eta|<1.8$. It is used to correct for fluctuations in the energy loss upstream of the calorimeter. Due to the position of the central solenoid coil inside the LAr barrel calorimeter, between the aluminum cryostat walls, the amount of material traversed by particles before reaching the electromagnetic calorimeter typically ranges between 2.5 and 6 radiation lengths (apart from a small transition region with higher values) depending on $\eta$. The ATLAS electromagnetic LAr calorimeter is characterized by a good energy resolution, impressive long-term stability, radiation resistance and uniformity. It's high granularity allows sophisticated particle identification and pile-up rejection.

\subsection{CMS}

The CMS electromagnetic calorimeter is a compact homogeneous calorimeter operating inside a $3.8 \mathrm{~T}$ superconducting solenoid $(6 \mathrm{~m}$ inner diameter), a detailed description can be found in Refs. [2], [4] and [5]. It is composed of Lead Tungstate $\left(\mathrm{PbWO}_{4}\right.$, in the following PWO) scintillating crystals organized in a barrel $\left(61200\right.$ crystals of approximately $\left.2.2 \times 2.2 \times 23 \mathrm{~cm}^{3}\right)$ covering the central pseudorapidity region $|\eta|<1.48$ and two endcaps (14648 crystals of approximately $3 \times 3 \times 22 \mathrm{~cm}^{3}$ ) which extend the coverage up to $|\eta|=3$. A pre-shower detector, a sampling calorimeter made of lead and silicon strips, which is placed in front of the endcaps, is meant to improve the $\gamma-\pi^{0}$ separation in this region. The light from the crystals is detected by avalanche photo diodes (APD) in the barrel and vacuum photo triodes (VPT) in the endcaps. The compactness and transverse granularity of the calorimeter $\Delta \eta \times \Delta \varphi=0.0175 \times 0.0175$ reflect the properties of PWO, a very dense $\left(\rho=8.28 \mathrm{~g} / \mathrm{cm}^{3}\right)$ scintillating material characterized by small radiation length $(0.89 \mathrm{~cm})$ and small Molière radius $(2.0 \mathrm{~cm})$. After an extensive R\&D phase, the PWO crystals produced are fast ( $80 \%$ of light is emitted within $25 \mathrm{ns)}$ ) and suffer a moderate loss of transparency [6] with radiation for fluences and dose rates originally foreseen at the LHC. These changes of signal necessitate a very stable transparency monitoring system in order to maintain the design energy resolution of $0.5 \%$ at high energies. With the whole calorimeter being inside the CMS solenoid, the material

\footnotetext{
${ }^{1}$ The pseudorapidity $\eta$ is defined in terms of the polar angle $\theta$ measured from the beam line as $\eta=-\ln \tan (\theta / 2)$.
} 
in front is only due to the tracker system, and ranges from 0.4 and 1.9 radiation lengths depending on the pseudorapidity. The calorimeter shows an excellent energy resolution and good uniformity, although corrections for radiation induced response fluctuations are necessary.

\section{Calibration}

In order to achieve the required performance, the response of the ATLAS and CMS calorimeters need to be monitored and calibrated periodically using specific calibration methods or physics channels. These calibration procedures are outlined in detail in Refs. [7] and [8] for ATLAS and CMS, respectively and summarized in this section.

\subsection{ATLAS}

The calibration of the electron and photon energy scales in ATLAS can be seen as a three-step process: The cell-level calibration of the read-out electronics, the MC-based cluster level calibration including dead-material and layer corrections obtained from data-MC comparisons of single particle showers (muons, unconverted photons and electrons) and the in-situ calibration using the invariant mass peak of $Z \rightarrow$ ee decays. The concept of LAr calorimeter electronics calibration is to inject a well known exponential pulse as close as possible to the point where the ionization pulse is usually created in the detector and read it back with the normal front-end electronics. In that way, electronics gain, pulse shapes and pedestals are extracted on a regular basis and updated in the database in case differences are detected with a typical update frequency of once per month. Due to its bipolar pulse shape, the in-time pile-up will be compensated in average by the out-of-time pile-up of preceeding bunch crossings, provided the bunch luminosities are equal. In real LHC fills, however, the machine is filled with bunch trains leading to empty bunches in between trains and unequal bunch luminosities. As such, the pedestal (baseline) will become a function of the position inside a train and the average pile-up density per bunch crossing. A cell-by-cell correction of this effect based on the exact knowledge of the bunch luminosities and the physics pulse-shapes is applied. This correction is shown in the left plot of Fig. 1. For a typical EM cluster $(\Delta \eta \times \Delta \varphi=0.013)$ and an average pile-up density of $\langle\mu\rangle=40$, the maximum baseline shift is $800 \mathrm{MeV}$, which is reduced to $<30 \mathrm{MeV}$ after correction. Layer corrections are needed in data to adjust for residual effects that are not perfectly accounted for by the cell-level electronics calibration. These layer corrections are extracted from data-MC comparisons of the response of muons, electrons, and unconverted photons. Muons are mostly insensitive to the material upstream, whereas electrons and unconverted photons allow the determination of the material upstream and the material between the PS and the accordion. In order to obtain energies of electrons and photons, the energies of cells in a region around a high energy deposit are summed into clusters (e.g. cluster size of $\Delta \eta \times \Delta \varphi=0.075 \times 0.175$ for electrons and converted photons in the barrel). However, a simple sum of cell energies is not sufficient since the energy lost upstream $(\approx 5$ to $15 \%$ ), the energy outside the cluster, and the energy behind the calorimeter (longitudinal leakage) needs to be corrected for. Instead, the corrected energy is obtained by means of a multivariate algorithm (MVA), trained with single particle MC events (target $\left.E_{\text {true }} / E_{\text {meas }}\right)$ with the following input variables: the energy in the accordion $E_{\text {acc }}$, the energy in the presampler $E_{\mathrm{PS}}, \eta, \varphi$, the shower-depth, and the shower-width (and extra variables for converted photons). This procedure is done independently for electrons, unconverted photons and converted 
photons. It is obvious that an accurate MC description of the calorimeter and the material in front of the calorimeter is essential for this approach. Finally the absolute energy scale is calibrated in $\eta$-bins using the invariant mass distribution of the $\mathrm{Z}$ boson (no $\varphi$ dependence). Decays of $\mathrm{J} / \Psi \rightarrow$ ee and $\mathrm{Z} \rightarrow \mathrm{ll} \gamma$ are used to check the validity of the calibration to lower energy electrons and to photons.

\subsection{CMS}

Due to the small Molière radius of the PWO crystals, $80 \%$ of the energy of the impinging electron or photon is deposited in a single crystal (for central incidence). The digitized signals ( $40 \mathrm{MHz}$ sampling rate) are reconstructed using the out-of-time pile-up resistant multifit algorithm which minimizes the $\chi^{2}$ between ten digitized samples and a linear combination of a possible signal in the triggered bunch crossing $A_{i}$ and nine out-of time pile-up signals. The reconstruction of the total energy of an electron or photon implies the summation of energy deposits in a cluster of crystals. Superclusters of clusters along $\varphi$, the bending direction, are formed dynamically before applying other corrections:

$$
E_{\mathrm{e}, \gamma}=F_{\mathrm{e}, \gamma} \cdot\left(G \cdot \sum_{i} C_{i} \cdot S_{i}(t) \cdot A_{i}+E_{\mathrm{ES}}\right),
$$

where the energy deposited in each crystal is given by the pulse amplitude $A_{i}$, in ADC counts, multiplied by ADC-to-GeV conversion factor $G$, measured separately for the barrel (EB) and endcaps (EE), by the intercalibration coefficients $C_{i}$ of the corresponding channel, and by $S_{i}(t)$, a correction term due to radiation-induced channel response changes at time $t$. The calibrated preshower energy $E_{\mathrm{ES}}$ is added to the endcap energy deposits. The factor $F_{\mathrm{e}, \gamma}$ represents the energy correction applied on the superclusters necessary to take into account the $\eta$ - and $\varphi$-dependent geometry and material effects as well as the fact that electrons and photons interact slightly differently in the calorimeter. It is obtained by an MVA, tuned on MC and maximally exploiting the information of the event. Again, an excellent data-MC agreement is the prerequisite. The relative variation of the channel response measured by the laser light injection, is shown in the right plot of Fig. 1 as a function of time for different $\eta$ regions. Correction factors $S_{i}(t)$, to correct for response changes, are calculated online and are available a few hours after having taken data. The corrected signal is restored and checked using $\pi^{0}$ decays and $\eta \rightarrow \gamma \gamma$. The laser system is itself monitored by PN diodes and is very stable in time. Once validated, the correction factors are inserted into a database and promptly-corrected data can be used for many physics analyses.

More refined offline corrections, on the other hand, are necessary to reach the best performance of the calorimeter. A number of methods is used to obtain the intercalibration coefficients $C_{i}$ from data: The $\varphi$-symmetry method is based on the expectation that, for a large sample of minimum bias events, the total deposited energy should be equal in all crystals at same $\eta$ ( $248 \eta$ rings of crystals). It is the fastest method but its accuracy is limited by systematics, mainly due to the fact that the upstream material (e.g. tracker layers and support structure) is not uniformly distributed in $\varphi$. The $\pi^{0}$ - and $\eta$-meson calibrations use the invariant mass of the photon pairs from these meson decays. Unconverted pairs of photons in $3 \times 3$ matrices of crystals are selected online as $\pi^{0} / \eta$ candidates. On top of that, the comparison of the energy measured in the calorimeter with the track momentum from the silicon tracker for isolated electrons from $\mathrm{W}$ - and $\mathrm{Z}$ boson decays is used to obtain the intercalibration constants. Inter-calibrations from the different methods are combined to 

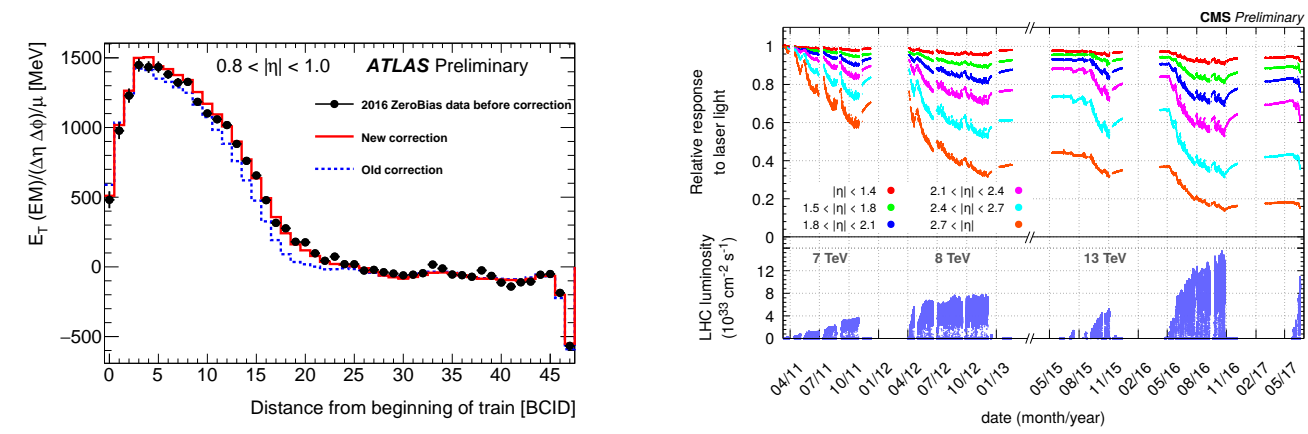

Figure 1. Left: Bunch crossing (BCID) dependent baseline correction in the ATLAS LAr calorimeter [9]. The plot compares the correction (blue and red lines) with zero bias data recorded in 2016. Right: Relative response of the CMS ECAL to laser light during 2011, 2012, 2015, 2016 and 2017 data taking [10]. An average is shown for each $\eta$ range. The bottom plot shows the corresponding instantaneous luminosity. The response change observed in the ECAL channels is up to $10 \%$ in the barrel and it reaches up to $50 \%$ at $\eta \sim 2.5$. The recovery of the crystal response during the periods without collisions is visible.

give the overall inter-calibration coefficients. The Run- 1 combined precision of the inter-calibration depended on $\eta$. In the central barrel $\eta \leq 1$ the precision obtained was $0.5 \%$, rising to $0.7 \%$ in the highest $\eta$ region of the barrel. In the endcaps the precision was ranging from $1 \%$ to $2 \%$ depending on the distribution of the material in front.

The final step in the calibration procedure is the calculation of the global energy scale. This is found by measuring the invariant mass peak from $\mathrm{Z}$ decays to dielectrons to correct the ADC-to-GeV scale factors for the EB and EE. The width of the peak is also a useful parameter for studying the calorimeter resolution, especially relevant for searches for particles around $100 \mathrm{GeV}$. Decays of $\mathrm{Z}$ bosons into two muons where one muon radiates a photon, $\mathrm{Z} \rightarrow \mu \mu \gamma$, are used to cross-check the energy calibration of photons.

\section{Resolution, Uniformity and Linearity}

The calorimeter resolution is usually parameterized as

$$
\frac{\sigma_{E}}{E}=\frac{a}{\sqrt{E}} \oplus \frac{b}{E} \oplus c,
$$

where $a$ is the stochastic term, $b$ the noise term and $c$ the constant term. Both experiments have determined the full resolution function in test beams. Stochastic terms of $a \sim 10 \%$ and $a \sim 3 \%$ have been measured for ATLAS and CMS respectively. Together with a noise term for a typical cluster of $b \sim 100$ to $200 \mathrm{MeV}$, and a local constant term of $c \sim 0.2 \%$ to $0.3 \%$ these values were measured at a test beam without magnetic field and with very little material upstream. On top of that, non-uniformities of the energy response as a function of $\eta$ and $\varphi$ and in time will increase this constant term, which can be expressed by an effective constant term $c_{\text {eff }}$ added in quadrature to $c$. For the typical energy range of electrons from $\mathrm{Z}$ decays $\left(E_{\mathrm{T}} \approx 30\right.$ to $\left.50 \mathrm{GeV}\right)$, only the stochastic term $a$ and the constant term $c$ are relevant. Since data-MC comparisons of the invariant mass peak of low-energetic electrons from $\mathrm{J} / \Psi$-decays $\left(E_{\mathrm{T}} \approx 5\right.$ to $\left.15 \mathrm{GeV}\right)$, for which the resolution is 
dominated by the stochastic and noise terms $a$ and $b$, showed good agreement ([7], [11], [8] and [12]), data-MC comparisons of the invariant mass distribution of electrons from $\mathrm{Z}$ decays can be used to directly determine $c_{\text {eff }}$. The left plot of Fig. 2 shows the extracted effective constant term for ATLAS using Run-1 data [7]. Similar results have been obtained with Run-2 data [11]. In the central barrel a constant term of $c_{\text {eff }} \approx 0.7 \%$ is obtained which is in good agreement with the measured azimuthal non-uniformity. The right plot [12] shows the measured and simulated resolution as a function of $\eta$ for electrons from $\mathrm{Z}$ decays in CMS for a category of electrons with low bremsstrahlung ( $>94 \%$ of the energy in the $3 \times 3$ central cells of the supercluster). In summary,
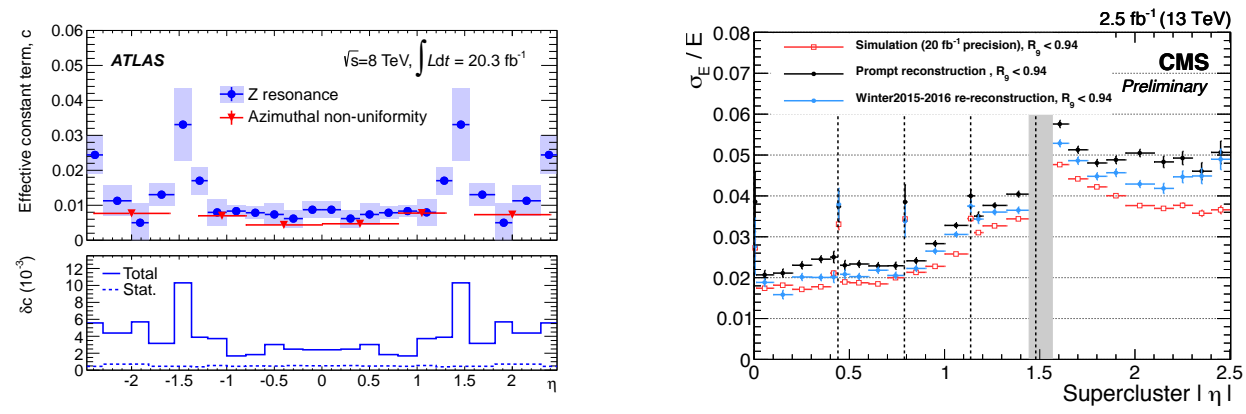

Figure 2. Right: Effective constant term $c_{\text {eff }}$ in ATLAS as a function of pseudorapidity extracted from the width of the $\mathrm{Z}$ peak [7]. Left: Comparison of simulated and measured resolution in the CMS ECAL for electrons from $\mathrm{Z}$ decays with low bremsstrahlung [12].

electrons from $\mathrm{Z}$ decays are measured with a resolution of $\approx 1.5$ to $2.1 \%$ in the central barrel of CMS electromagnetic calorimeter with a strong dependence on bremsstrahlung. Compared to that, the ATLAS electromagnetic calorimeter obtains a resolution of $\approx 1.7$ to $1.9 \%$ in the same region. For both calorimeters the energy resolution rapidly degrades with higher pseudorapidity. It should be noted, that despite very different stochastic terms, the two calorimeters perform similarly in the central barrel region, although for the best category CMS clearly outperforms ATLAS. Both calorimeters have measured a linearity of per-mille level precision between 20 and $60 \mathrm{GeV}$ ([7] and $[8])$.

\section{Performance in Selected Physics Channels}

In this section the Higgs boson mass measurement will be used to compare the performance of the two electromagnetic calorimeters. It is remarkable, that despite the totally different conceptions of these two LHC experiments, in July 2012 the discovery of the Higgs boson ([13] and [14]) were announced by both collaborations with very similar overall significances. In 2015 a combined measurement of the Higgs boson mass was published [15], which demonstrates well the strengths and weaknesses of both calorimeters. Here, I will concentrate on the most relevant decay channel for the electromagnetic calorimeters, $\mathrm{H} \rightarrow \gamma \gamma$. As shown in Ref. [15], the systematic uncertainties on the Higgs mass are dominated by the energy and momentum scale uncertainties in both experiments. The total uncertainty is $0.27 \mathrm{GeV}$ and $0.15 \mathrm{GeV}$ for ATLAS and CMS respectively. ATLAS attributes larger uncertainties to imperfect knowledge of the material in front of the calorimeter $(0.15 \mathrm{GeV}$ vs $0.07 \mathrm{GeV}$ ), which can be understood by the fact that due to the higher material budget, any additional 
material has a $\sim 2 \times$ larger impact in ATLAS than in CMS. Also other uncertainties in the lateral shower shape or longitudinal response of the calorimeter are larger in ATLAS which is partly due to a slightly poorer data-MC agreement of shower shapes in ATLAS. The statistical uncertainty is $0.43 \mathrm{GeV}$ and $0.31 \mathrm{GeV}$ in ATLAS and CMS respectively. Even though the inclusive $\mathrm{H} \rightarrow \gamma \gamma$ mass peak shows a similar width in both experiments, the statistical error is dominated by the best category, in which CMS outperforms ATLAS. However, ATLAS compensates the worse stochastic term of the calorimeter resolution almost entirely by the photon pointing which helps to determine the correct primary vertex.

Recently a measurement of the W-mass using the electron and muon decay channels was published by ATLAS [16]. The obtained mass value is $m_{\mathrm{W}}=80370 \pm 19 \mathrm{MeV}$, with a remarkably small uncertainty that is comparable to Tevatron measurements. For such a high-precision measurement, the stability of the ATLAS electromagnetic calorimeter is clearly an advantage since the full statistics could be used to reduce the systematic errors.

\section{Upgrade Plans - Calibration Strategies}

At the HL-LHC, instantaneous luminosities of up to $\mathcal{L}=7.5 \times 10^{34} \mathrm{~cm}^{-2} \mathrm{~s}^{-1}$ are expected, resulting in an average of $\langle\mu\rangle=200$ proton collisions per bunch crossing. One challenge for HL-LHC therefore will consist of selecting and isolating interesting hard scatter collisions from the majority of soft pile-up collisions. Therefore, in addition to improving radiation hardness and increasing trigger and read-out rates, in their planned upgrades, both experiments mainly focus on improving their pile-up rejection capabilities ([17], [18] and [19]). One clear improvement in both experiments will come from an extension of the inner tracker up to $|\eta|<4$. On top of that timing information obtained from dedicated timing detectors located between tracker and calorimeter (CMS: $|\eta|<3$, ATLAS: $2.4<|\eta|<4.0$ ) with a timing resolution for minimum ionizing particles (MIPs) of $\sigma_{t} \approx 30 \mathrm{ps}$ will supplement the position information provided by the trackers. Both calorimeters will improve the granularity of the hardware trigger and CMS is planning to replace the endcap calorimeter $(1.5<|\eta|<3.0)$ by a High Granularity Calorimeter (HGCal) which will allow 3D imaging of particle showers and hence yield a wealth of information that can be used for pile-up mitigation. In the following, this calorimeter is described in a nutshell and the planned calibration strategy briefly outlined.

The HGCal is a sampling calorimeter with silicon and plastic scintillator as the active materials. It includes both electromagnetic (CE-E) and hadronic (CE-H) sections. The former will use lead as the main absorber material and hexagonal silicon sensors as the active detector. The CE-H will use stainless steel as absorber and a mixture of silicon (in the regions of highest radiation) and scintillator as detectors. In total six million silicon channels will have to be calibrated regularly since their response will not be constant due to radiation damage. The goal will be to keep the constant term $c$ below $1 \%$. In order to achieve that, the contribution of non-uniformities of the silicon response to the constant term should be limited to $<0.5 \%$. Simulations have shown [20], that an inter-calibration of the silicon channels with a precision of $<3 \%$ is necessary to keep the contribution to the constant term below $0.5 \%$. The number of minimum ionizing particles needed to achieve this precision depends on the signal-to-noise ratio $S / N$. For a $S / N>3$ about 1.5 million events are sufficient to obtain an inter-calibration with the required precision. However, for lower 
$S / N$ ratios of $\approx 2$ which are expected due to the radiation damage after an integrated luminosity of $3 \mathrm{ab}^{-1}$ up to 100 times larger statistics will be needed, which will be accumulated using the full level-1 trigger sample by building histograms in the high level trigger. Different ways of rejecting noise hits by requiring tracks have been successfully attempted ("tracking procedure", see Ref. [21]). In test beams at CERN and FNAL [20] it was demonstrated that the channel calibration constants could be obtained with the necessary precision by using fits of Landau distributions convoluted with Gaussians to the energy deposit distribution from minimum ionizing particles. The left plot of Fig. 3 shows the MIP peak from test beam measurements of one typical channel with a S/N ratio of 7.69 , in which a precision on the peak of $\approx 0.3 \%$ was obtained. The right plot depicts the MIP signal for all calibrated cells as a function of the interim read-out chip Skiroc2 used for the 2016 test beam campaigns [20]. These test beam results are very encouraging, but it will be difficult and will probably take some time to obtain such precise inter-calibration results in situ.

On top of the time-dependent inter calibration using MIP signals, an independent monitoring of the linearity will be performed using a charge injection system.
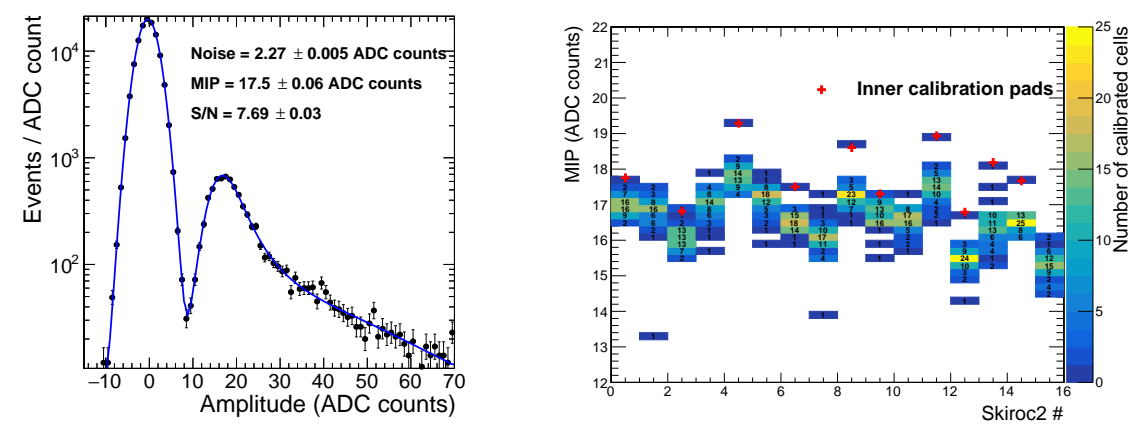

Figure 3. Left: Amplitude spectrum, measured in ADC counts, of signals in a typical silicon cell in 2016 from incident charged pions in the CE-E section. The amplitude is fitted with a sum of a Gaussian (for the pedestal) and a convolution of a Landau distribution and a Gaussian (for the signal). Right: MIP signal for all calibrated cells, as a function of the read-out chip Skiroc2, for the CERN tests in 2016 [20].

\section{Conclusions}

The electromagnetic calorimeters of ATLAS and CMS and their respective calibration strategies have been described. Their performances measured during LHC collisions data taking have been compared and discussed. It has been shown, that both calorimeters achieve a resolution, uniformity, and linearity which is very close to the design values and fully within the requirements dictated by benchmark physics channels. These results prove that the calibration strategies applied are sound and robust, even at high luminosities. The different strengths and weaknesses of the ATLAS and CMS electromagnetic calorimeters have been briefly discussed on the basis of the Higgs mass and the $\mathrm{W}$ mass measurements. Both experiments are planning to upgrade their detectors for HL-LHC. The calibration strategy that is currently planned for the CMS HGCal has been described and first test beam measurements demonstrating that the required inter-calibration precision can be achieved have been shown. 


\section{Acknowledgements}

We thank CERN for the very successful operation of the LHC, as well as the support staff from our institutions without whom ATLAS and CMS could not be operated efficiently.

\section{References}

[1] ATLAS Collaboration, The ATLAS experiment at the CERN LHC, J. Instrum., 3 S08003 (2008)

[2] CMS Collaboration, The CMS experiment at the CERN LHC, J. Instrum $3 \mathrm{~S} 08004$ (2008)

[3] ATLAS Collaboration, Liquid Argon Calorimeter Technical Design Report, CERN/LHCC/96-041

[4] CMS Collaboration, The Electromagnetic Calorimeter Technical Design Report, CERN/LHCC/97-33

[5] CMS Collaboration, Performance and operation of the CMS electromagnetic calorimeter, J. Instrum 5 T03010 (2010)

[6] P. Adzic et al., Radiation hardness qualification of PbWO4 scintillation crystals for the CMS Electromagnetic Calorimeter, JINST 5 (2010) P03010

[7] ATLAS Collaboration, Electron and photon energy calibration with the ATLAS detector using LHC Run 1 data, Eur. Phys. J. C 74 (2014) no.10, 3071

[8] CMS Collaboration, Energy Calibration and Resolution of the CMS Electromagnetic Calorimeter in pp Collisions at $\sqrt{s}=7 \mathrm{TeV}$, JINST 8 (2013) P09009

[9] ATLAS Collaboration, ATLAS LAr public plots, https://twiki.cern.ch/twiki/bin/view/AtlasPublic/ApprovedPlotsLAr

[10] CMS Collaboration, CMS ECAL Laser monitoring up to 2017, $\pi^{0} / \eta \rightarrow \gamma \gamma$ spectrum and monitoring, ES calibration, CMS DP-2017/023 (2017)

[11] ATLAS Collaboration, e/gamma calibration using $\sqrt{s}=13 \mathrm{TeV}$ data from 2015-2016, ATL-PHYS-PUB-2016-015

[12] CMS Collaboration, 2015 ECAL detector performance plots, CMS-DP-2015/057

[13] CMS Collaboration, Observation of a new boson at a mass of $125 \mathrm{GeV}$ with the CMS experiment at the LHC, Phys. Lett. B716 (2012)

[14] ATLAS Collaboration, Observation of a new particle in the search for the Standard Model Higgs boson with the ATLAS detector at the LHC, Phys. Lett. B716 (2012)

[15] ATLAS and CMS Collaborations, Combined Measurement of the Higgs Boson Mass in pp Collisions at $\sqrt{s}=7$ and $8 \mathrm{TeV}$ with the ATLAS and CMS Experiments, Phys. Rev. Lett. 114, 191803 (2015)

[16] ATLAS Collaboration, Measurement of the W-boson mass in pp collisions at $\sqrt{s}=7 \mathrm{TeV}$ with the ATLAS detector, arXiv:1701.07240 [hep-ex] (2017)

[17] ATLAS Collaboration, ATLAS Phase-II Upgrade Scoping Document, CERN-LHCC-2015-020

[18] CMS Collaboration, CMS Phase-II Upgrade Scope Document, CERN-LHCC-2015-019

[19] CMS Collaboration, Technical Proposal for the Phase-II Upgrade of the Compact Muon Solenoid, CERN-LHCC-2015-10

[20] CMS Collaboration, CMS High Granularity Endcap Calorimeter Technical Design Report, being published

[21] CMS Collaboration, First beam tests of prototype silicon modules for the CMS High Granularity Endcap Calorimeter, CMS DN-17-011, submitted to JINST 